\title{
Psicooncología
}

ISSN: 1696-7240

\section{Competencia para el cuidado en el hogar diada persona con cáncer en quimioterapia-cuidador familiar}

\author{
Laura Milena Sánchez ${ }^{1 *}$; Gloria Mabel Carrillo²
}

Recibido: 15 de marzo de 2017 / Aceptado: 15 de abril de 2017

Resumen. Objetivo: Determinar la relación entre el nivel de competencia para el cuidado en el hogar de la diada persona con cáncer en quimioterapia y su cuidador familiar. Método: estudio cuantitativo, descriptivo, correlacional de corte transversal. Participaron 80 diadas que asistieron a los servicios de Hemato-oncología en Floridablanca (Colombia). Se utilizó la ficha de caracterización de la Diada cuidador-persona con enfermedad crónica y competencia para el cuidado en el hogar de la persona con enfermedad crónica/cuidador familiar. Resultados: Las diadas de cuidado en su mayoría son mujeres, de estratos socioeconómicos medios, con niveles de escolaridad medios; ocupación hogar y jubilados. La competencia para el cuidado en el hogar de la diada en general y por dimensiones presenta puntajes medios, siendo ligeramente más baja en las personas con cáncer en quimioterapia respecto a los cuidadores. Se identificó una correlación débil sin ser estadísticamente significativa entre la competencia de la persona con cáncer y su cuidador familiar $(\mathrm{p}=0,067)$. Sin embargo, en las dimensiones conocimiento, disfrutar, anticipación y relación e interacción social se evidenciarelación positiva y estadísticamente significativa. Conclusión: La competencia para el cuidado en el hogar es un fenómeno central a intervenir en el cuidado de enfermería durante la transición hospital-hogar y en la generación de planes de alta que aseguren la continuidad del tratamiento.

Palabras claves: Competencia; cuidador familiar; neoplasias; alta del paciente.

\section{[en] Competency for homecare of that dyad person with cancer in chemotherapy and his caregiver family}

\footnotetext{
Abstract. Objective: To determine the relationship between the level of competence for home care of the person with cancer in chemotherapy and their family caregiver. Method: quantitative, descriptive, correlational cross-sectional study. 80 dyads attended the services of Hematooncology in Floridablanca (Colombia). The characterization form of the Caregiver-person with chronic illness and the instrument of competency for homecare were used. Results: The caregivers and the patients with chronic illness are mostly women, from middle socioeconomic strata, with average schooling levels; Household and retired occupations. The competency for the home care of the dyad in general and for the dimensions has average scores, being slightly

1 M. Enfermera. Magister en Enfermería. Enfermera hemato-oncología Clínica FOSCAL-Floridablanca Santander Colombia.

E-mail: lamsanchezma@unal.edu.co

2 Universidad Nacional de Colombia. Facultad de Enfermería sede Bogotá.

E-mail: gmcarrillog@unal.edu.co

* Dirección de correspondencia: Gloria Mabel Carrillo. Universidad Nacional de Colombia. Sede Bogotá. Facultad de Enfermería.

E-mail.gmcarrillog@unal.edu.co
} 
lower in people with cancer than in their caregivers. A weak relationship was identified without being statistically significant between the competency of the person with the cancer and their family caregiver $(p=0.067)$. However, in the dimensions of knowledge, enjoyment, anticipation and relationship and social interaction there are positive and statistically significant relationship. Conclusion: Competency for home care is a central phenomenon to intervene in nursing care during the hospital-home transition and in the generation of discharge plans that ensure continuity of treatment.

Key words: Competence; family caregiver; neoplasm; patient discharge.

Sumario. 1. Introducción 2. Método 3. Resultados 3.1. Perfil y caracterización de los participantes 3.2. Cuidadores familiares de personas con cáncer en tratamiento de quimioterapia: 3.3. Percepción de carga y apoyo 3.4. Competencia para el cuidado en el hogar 4. Discusión 5. Referencias bibliográficas.

Cómo citar: Sánchez LM, Carrillo GM. Competencia para el cuidado en el hogar diada persona con cáncer en quimioterapia-cuidador familiar Psicooncología 2017;14: 137-48. DOI: 10.5209/PSIC.55817

\section{Introducción}

El cáncer condiciona un curso de enfermedad natural prolongado con cambios desde el diagnóstico de la persona enferma y sus cuidadores en múltiples aspectos de la vida diaria y que incluyen las esferas social, familiar, económica, física y psicológica. Los organismos gubernamentales han descrito el notable crecimiento que tienen las enfermedades crónicas no transmisibles como el cáncer con más de 11 millones de casos nuevos al año ${ }^{(1)}$, sus repercusiones en los individuos enfermos- cuidadores y sobre los sistemas de seguridad social que cada vez demandan y reclaman intervenciones estratégicas que disminuyan costos y aminoren el impacto que estas generan. En el abordaje de las situaciones que se pueden presentar a lo largo del proceso de diagnóstico, inicio de tratamiento y egreso se hace relevante explorar el vínculo que se establece entre el paciente y su cuidador familiar y la conformación de un nuevo sujeto de cuidado denominado "diada" que debe afrontar el seguimiento de la terapia oncológica, adquiere responsabilidades para el cuidado diario en actividades básicas e instrumentales y toma decisiones inherentes al manejo de la condición de salud-enfermedad, entre otros aspectos ${ }^{(2,3)}$. Ahora bien, ante una terapia oncológica como la quimioterapia, estas diadas experimentan un sin número de situaciones estresantes ligadas al imaginario social que se tiene de este tipo de tratamiento, al manejo de efectos secundarios, la duración y los cambios en los estilos de vida con afectación de los diferentes niveles de bienestar ${ }^{(2,4)}$.

La diada está conformada por las personas con enfermedad crónica y sus cuidadores, que generan un vínculo estrecho y por tanto demandan mucho tiempo de relación generando gran impacto a nivel actitudinal, familiar, cultural, espiritual, económico, forma de vida y en calidad de vida que hacen necesario que el cuidado no se entienda aisladamente sino como un binomio ${ }^{(5)}$. Las competencias que han de adquirir las diadas para el cuidado en este proceso son insumo fundamental para garantizar la continuidad del tratamiento, disminuir las complicaciones, los reingresos por factores prevenibles y minimizar la sobrecarga del cuidador $^{(6)}$. En tal sentido, el abordaje de esta diada demanda estrategias específicas en los planes de alta y egreso por parte de enfermería para ofrecer cuidados acordes a las necesidades en particular. 
El estudio de las competencias para el cuidado en el hogar de la diada se debe generar desde una perspectiva integral en la que se identifiquen aspectos de conocimiento de la enfermedad, condiciones personales, habilidades instrumentales, capacidad de anticiparse, factores básicos de bienestar y disfrutar, interacción social y redes de apoyo ${ }^{(7)}$, es decir, con indicadores útiles para las instituciones de salud que aseguren un cuidado continuo y de calidad una vez egresen los usuarios de los servicios. Así mismo, es imprescindible contar con un diagnóstico de las competencias de las diadas que se constituya en insumo central para la consolidación de intervenciones y a la vez, en indicador de proceso y resultado que facilite la identificación del impacto de estas intervenciones. Es de resaltar que en el contexto colombiano no se han documentado estas competencias en pacientes que se encuentran en tratamiento de quimioterapia ni desde la perspectiva de la diada enfermocuidador familiar.

De acuerdo con lo anterior, el objetivo del estudio fue describir y determinar la relación entre el nivel de competencia para el cuidado en el hogar de la diada persona con cáncer en tratamiento con quimioterapia y su cuidador familiar atendidos en los servicios de hematooncologia de una institución de cuarto nivel del municipio de Floridablanca (Santander), centro de referencia regional en la atención de personas con cáncer.

\section{Método}

Estudio de abordaje cuantitativo, descriptivo, correlacional, de corte transversal realizado en el 2016. La población elegible estuvo constituida por las personas con cáncer y sus cuidadores familiares, en los servicios de hospitalización de hemato-oncología y ambulatorios.

Fueron criterios de inclusión: para la persona con cáncer: mayor de 18 años, con diagnóstico de cáncer en tratamiento de quimioterapia.

Para el cuidador familiar: persona con vínculo de cercanía o parentesco a cargo del cuidado de un enfermo con cáncer que se encuentre en tratamiento con quimioterapia, mayor de 18 años, con un tiempo mayor o igual a 3 meses de labor como cuidador familiar.

Se excluyeron personas con cáncer neurológicamente no conectadas con el medio, o con trastorno mental.

Se revisaron 142 historias clínicas de personas que asistían a los servicios en el periodo del estudio a recibir quimioterapia, considerados la población potencial. Se descartaron 61 por no cumplir el criterio de inclusión de ser una diada ya que los pacientes no contaban con un cuidador familiar (52 casos), o estos no tenían un tiempo como cuidador mayor a 3 meses (10 casos). La muestra final se conformó con 80 diadas de pacientes con cáncer en quimioterapia y su cuidador familiar.

Los instrumentos utilizados fueron: "la ficha de caracterización de la diada Cuidador Persona con enfermedad crónica" ${ }^{(8)}$ que contiene 42 ítems organizados en 3 categorías: 1. Condiciones del paciente y su perfil sociodemográfico; 2. percepción de carga y de apoyo 3. Medios de información y comunicación con los que la diada cuenta y "Competencia para el cuidado-cuidador" el GCPC-UN-CPC(7) que consta de 60 ítems que se miden con una escala tipo Likert con puntuaciones que van de 1 a $4 ; 1$ es nunca o casi nunca, 2 pocas veces, 3 con frecuencia y 4 casi siempre o siempre. Contiene 6 categorías que se ordenan bajo el acróstico CUIDAR e incluyen: Conocimiento (nociones e ideas respecto a la enfermedad, las terapéuticas farmacológicas y no farmacológicas, las indicaciones especiales de la terapéutica, el manejo de dispositivos y equipos durante el regreso del paciente). Unicidad (características de personalidad, base fundamental para que se afronte la enfermedad). 
Instrumental: (habilidades y destrezas, así como las competencias para realizar las labores de cuidado). Disfrutar (bienestar): referida como el grado de bienestar que se tiene para comprender cómo se asume la responsabilidad del cuidado en casa. Anticipación (nociones e ideas que se tienen, pensando en anticiparse a necesidades futuras) y Relación social e interacción: (redes de apoyo primarias y secundarias que se tienen y hacen parte del diario vivir). Esta escala cuenta con propiedades psicométricas para su uso en el contexto colombiano $^{(9,10)}$.

Los procedimientos del estudio fueron acordes a los principios establecidos en la Declaración de Helsinki ${ }^{(11)}$, las Pautas Éticas CIOMS ${ }^{(12,13)}$, y las normas científicas, técnicas y administrativas para la investigación en salud de la Resolución 8430 del 04 de Octubre de 1993 del Ministerio de Salud y Protección Social de la República de Colombia ${ }^{(14)}$ y a la Ley 911 de octubre 5 de 2004(15) "por la cual se dictan disposiciones en materia de responsabilidad deontológica para el ejercicio de la profesión de enfermería en Colombia"(16).

Se obtuvo aval de Comité de Ética de la Facultad de Enfermería de la Universidad Nacional de Colombia y del Comité de Investigación de la institución de salud correspondiente. Se aplicó el procedimiento de consentimiento informado.

\section{Resultados}

\subsection{Perfil y caracterización de los participantes}

La mayoría de los participantes son de género femenino $(63,7 \%)$ con edades entre los 19 y 83 años, media de 51,8 años. El 98,8\% presenta baja dependencia, seguido por el 1,3\%.

En cuanto al diagnóstico médico, el cáncer de mama ocupa el primer lugar representando el 23,8\% de los casos, seguido de leucemia linfoblástica aguda 13,8\%, linfoma Hodgkin $13,8 \%$, mieloma múltiple con $12,5 \%$, linfoma no Hodgkin $10 \%$, cáncer de ovario 3,8\%, y de recto 3,8\%. El 73,7\% de los pacientes se encontraba en tratamiento en fase de mantenimiento, seguido del 7,5\% que recibía quimioterapia de acondicionamiento, en menor proporción rescate, paliativos, consolidativos y neoadyuvante. La mayor parte son administrados por vía endovenosa en el $95 \%$ de los casos y cerca del 50\% en el ámbito intrahospitalario (Tabla 1). Los pacientes llevan con la enfermedad entre los 0 a 1 año: 70,2\%, 1 a 2 años: 13,9\%, 2 a 3 años: $3,8 \%, 3$ a 6 años: $12,7 \%$.

En la escolaridad, la mayoría tiene bachillerato $(38,8 \%)$, la ocupación más frecuente es pensionado y pertenecen al estrato socioeconómico 2 (32,5\%), la mayor parte de los pacientes reside en área urbana. Predomina la religión católica.

\subsection{Cuidadores familiares de personas con cáncer en tratamiento de quimioterapia}

La edad promedio es de 45,9, con un mínimo de 18 años y un máximo de 79 años, en su mayoría son del género femenino (72,5\%). Respecto a la escolaridad predomina el bachillerato, estado civil casado o por unión libre (66,3\%) y religión católica.

El 82,5\% de los cuidadores son procedentes del Departamento de Santander, El 93,0\% reside en zona urbana. La dedicación al cuidado está entre las 3 y 24 horas, con una media de 15,33 horas al día. 
Tabla 1. Perfil Sociodemográfico de la diada persona con cáncer en tratamiento de quimioterapia-Cuidador familiar.

\begin{tabular}{|c|c|c|c|}
\hline \multicolumn{2}{|l|}{ VARIABLE } & $\begin{array}{c}\text { PERSONA CON } \\
\text { CÁNCER } \\
\mathbf{n}=\mathbf{8 0}(\%)\end{array}$ & $\begin{array}{c}\text { CUIDADOR } \\
\text { FAMILIAR } \\
n=80(\%)\end{array}$ \\
\hline \multirow{2}{*}{ Género } & Femenino & 63,7 & 72,5 \\
\hline & Masculino & 36,3 & 27,5 \\
\hline \multirow{5}{*}{ Grado escolaridad } & Analfabeta & 2,5 & 1,3 \\
\hline & Bachiller & 38,8 & 41,3 \\
\hline & Primaria & 26,3 & 21,3 \\
\hline & Profesional & 21,3 & 20 \\
\hline & Tecnologo & 11,3 & 16,3 \\
\hline \multirow{2}{*}{ Zona } & Rural & 3,8 & 6,3 \\
\hline & Urbana & 96,3 & 93,8 \\
\hline \multirow{5}{*}{ Estado civil } & Casado & 48,8 & 50 \\
\hline & Separado & 6,3 & 7,5 \\
\hline & Soltero & 23,8 & 23,8 \\
\hline & Unión libre & 16,3 & 16,3 \\
\hline & Viudo & 5 & 2,5 \\
\hline \multirow{6}{*}{ Ocupación } & Empleado & 20 & 21,3 \\
\hline & Estudiante & 2,5 & 6,3 \\
\hline & Hogar & 31,3 & 33,8 \\
\hline & Independiente & 11,3 & 23,8 \\
\hline & Pensionado & 32,5 & 8,8 \\
\hline & Otros & 2,5 & 6 \\
\hline \multirow{6}{*}{ Estrato socioeconómico } & 1 & 13,8 & 12,5 \\
\hline & 2 & 32,5 & 31,3 \\
\hline & 3 & 25 & 25 \\
\hline & 4 & 18,8 & 22,5 \\
\hline & 5 & 3,8 & 3,8 \\
\hline & 6 & 6,3 & 5 \\
\hline \multirow{3}{*}{ Religión } & Católica & 81,3 & 75 \\
\hline & Cristiana & 13,8 & 20 \\
\hline & Otras & 5,2 & 5,2 \\
\hline
\end{tabular}

Fuente: Datos del estudio, 2016. 


\subsection{Percepción de carga y apoyo}

En las diadas, el parentesco más frecuente de la persona con cáncer y su cuidador es esposa (o) con un $42,5 \%$, seguido de su hijo(a) con $21,3 \%$ y en menor proporción padres, hermano (a) y otros con $11,3 \%$ y $10 \%$ respectivamente.

La mayoría de los cuidadores cuida a la persona con cáncer desde el momento del diagnóstico en el 96,3\% de los casos, y el 47,5\% de estos son los únicos cuidadores de la persona con cáncer. El $57,5 \%$ de los pacientes percibe que son una carga baja para sus familiares, seguido de los que perciben que son una carga moderada $(26,3 \%)$, alta (15\%) y muy alta $(1,3 \%)$.

Cuentan con diferentes apoyos para el cuidado de su familiar: Psicológico, familiar, religioso, económico y social, siendo la familia el apoyo más importante en un 96,3\% de los casos, seguido de apoyo religioso y económico en un $92,5 \%$, social en un $80 \%$ y finalmente el apoyo psicológico en $61,3 \%$.

El 78,8\% de las diadas refiere que utiliza las Tecnologías de información y comunicación (TIC) para apoyarse en el cuidado, con un nivel medio de apoyo percibido por estas $(50 \%)$. Por orden de preferencia para recibir información acerca del cuidado de su familiar se encuentra el televisor en un $26,3 \%$, seguido de Teléfono $10 \%$, computador $10 \%$ y finalmente el radio con 7,5\%. En cuanto a la apropiación de las TIC para el cuidado, calculada a partir de la valoración del conocimiento, acceso y uso del televisor, radio, computador, teléfono e internet, con puntajes posibles de 0 a 100 se identificó en primer lugar el televisor (media 67,65; (DT 33,32), seguido por el teléfono (media 54,53; DT 36,88), y el radio (media 36,64; DT 28,08). Los medios de menor apropiación son el computador (media 31,59; DT 34,4), y el internet (media 40,2; DT 41,1).

\subsection{Competencia para el cuidado en el hogar}

El nivel de competencia del cuidador familiar de persona con cáncer arrojó una media de 149,4, una DT de 19,89, mínimo (min.) de 93 y máximo (máx) de 180. Por rangos se obtuvo un nivel de competencia medio para esta población. En el paciente la media fue de 144,27 DT 24,86, mín de 58 y máx de 180, nivel de competencia medio. En cuanto a las dimensiones, los puntajes obtenidos fueron similares en las diadas, con tendencia al nivel medio, siendo ligeramente más altos los promedios en los cuidadores (Tabla 2).

Al comparar la competencia para el cuidado en el hogar (CUIDAR) de la persona con cáncer en quimioterapia y el cuidador familiar, mediante la prueba no paramétrica de U ManWhitney se identifica que estas son similares en general y en las dimensiones conocimiento, unicidad, instrumental, anticipación y relación/interacción. La dimensión disfrutar es diferente.

Al determinar si existe relación entre la competencia para el cuidado en el hogar de la persona con cáncer en tratamiento de quimioterapia y su cuidador familiar, mediante el coeficiente de correlación de Spearman se identifica que esta es débil $(0,206)$ sin ser estadísticamente significativa ( $\mathrm{p}=0,067)$.

En cuanto a las dimensiones existe relación estadísticamente significativa entre: el conocimiento del paciente y el conocimiento del cuidador $(0,244)(\mathrm{p}=0.029)$; la competencia para el cuidado en el hogar del paciente y la dimensión bienestar del cuidador familiar $(\mathrm{p}=$ $0,008)$; la dimensión disfrutar del paciente y disfrutar del cuidador $(\mathrm{p}=0,027)$; la anticipación del paciente y el disfrutar del cuidador $(\mathrm{p}=0,09)$; la relación/interacción del paciente y el disfrutar del cuidador (p 0,08) (Tabla 3). 


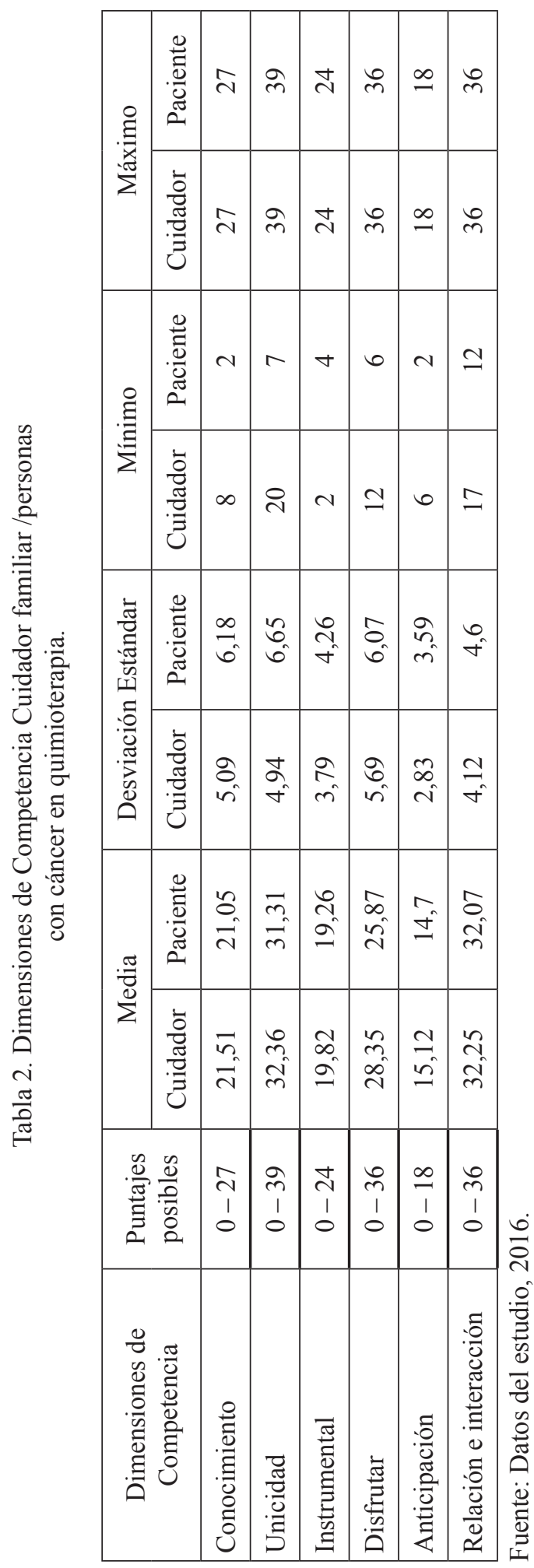




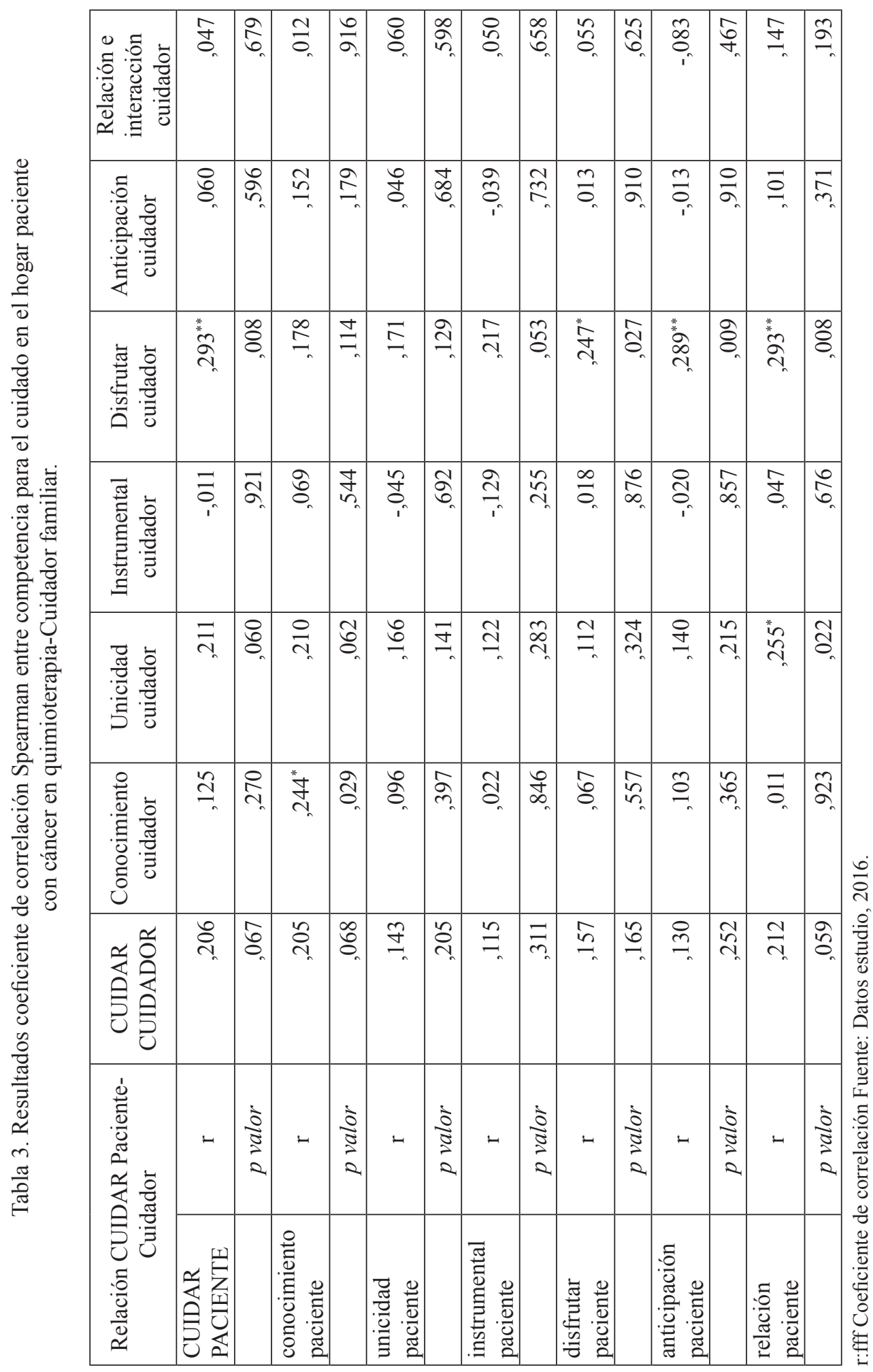




\section{Discusión}

En cuanto al perfil los hallazgos de género y estado civil de los pacientes son similares, a lo identificado en otro estudio en el municipio de Santander en población con enfermedad crónica oncológica ${ }^{(17)}$. En el estrato socioeconómico, escolaridad y la ocupación el estudio difiere a lo encontrado en otros ${ }^{(17,18)}$ en los que predomina estrato bajo, con muy bajos niveles de escolaridad y de ocupación hogar hallazgos relacionados con las características y tipo de usuario que se atiende en la Institución de Salud, predominan cotizantes, pensionados o beneficiarios dentro de un grupo familiar.

En los cuidadores familiares de personas con cáncer en tratamiento con quimioterapia el género femenino predomina, relacionado con la implicación cultural que se le ha dado al cuidado en donde las mujeres cumpliendo sus labores de madres, esposas e hijas asumen el rol de cuidar del enfermo ${ }^{(18-23)}$.

Los hallazgos descritos para los cuidadores son similares a otros estudios en el área metropolitana que reflejan predominio de estratos socioeconómicos bajos 1 y 2 . La ocupación más frecuente fue el hogar, lo cual está relacionado con el vínculo establecido y el tiempo que dedican a su labor, lo que se asemeja a lo encontrado por Avila ${ }^{(24)}$.

$\mathrm{Al}$ igual que el estudio de Puerto ${ }^{(19)}$ el estado civil más frecuente fue casado o en unión libre. A nivel nacional, Carrillo et al..$^{(9)}$, reporta que la mayor parte de cuidadores cuentan con un vínculo marital establecido en la región Andina, Caribe y Orinoquia y los solteros en Amazonia y Pacifica lo cual podría estar relacionado con el factor cultural propio de estas regiones. Predomina el grado de escolaridad bachiller lo cual se asocia en posibilidad para asumir el rol del cuidador ${ }^{(20,25)}$. La mayoría de los cuidadores cuidan a su familiar desde el momento del diagnóstico y casi la mitad son los únicos cuidadores, siendo estos hallazgos similares a otros estudios desarrollados en el área metropolitana de Bucaramanga ${ }^{(19,24)}$. El nivel de bienestar encontrado en los cuidadores de personas con cáncer en quimioterapia es alto en todas las esferas, lo cual podría estar asociado con el alto nivel de apoyo y variables propias de las personas enfermas a las que cuidan como alta funcionalidad, baja dependencia y asistencia por consulta externa a sus tratamientos activos contra la enfermedad que pueden llevar a que aún no se reconozca el impacto y carga que representa el cuidado y su afectación sobre los diferentes niveles de bienestar.

En el caso de los pacientes, se consideran una carga baja para la familia en más de la mitad de los casos, relacionado con la baja dependencia que tienen para el desarrollo de sus actividades diarias. En general predomina una alta percepción de bienestar físico, emocional, espiritual y social en los pacientes con cáncer pese a su enfermedad y el proceso que viven a diario con el tratamiento y los efectos adversos que estos traen, factores que se potencian en la medida que se administran más ciclos de quimioterapia y por tanto tienden a reducir niveles de bienestar ${ }^{(26)}$.

El uso de las TIC se consideran herramientas útiles que fortalecen el vínculo entre la diada y permiten un mejor desempeño en el rol de cuidador, tal como lo evidenció el estudio de la caracterización del cuidado de la diada entre cuidador familiar y persona con enfermedad crónica en donde el apoyo social con uso de TIC generó efectos positivos ${ }^{(8)}$. En el presente estudio; más del 50\% de las diadas hacen uso de ellas, siendo el nivel de apoyo percibido a través de ellas medio-alto, hallazgos que contrastan con otro estudio desarrollado en el 2014 en donde el nivel de conocimiento de las personas con enfermedad crónica frente al uso de las TIC fue de 85,1 en un nivel medio o bajo, siendo insuficiente el acceso a las mismas ${ }^{(7)}$. Finalmente, al evaluar el nivel de apropiación para cada una de las TIC se considera que el más importante es el televisor siendo la más pertinente para recibir información respecto al tratamiento de la enfermedad, cuidado y apoyo de la diada. 
En cuanto a la competencia para el cuidado en el hogar, los hallazgos se relacionan con lo identificado en un estudio previo a nivel nacional en pacientes con enfermedades crónicas y cuidadores familiares; predominan niveles medios de competencia en general y por dimensiones, siendo muy bajo el porcentaje con niveles óptimos esperados (altos) ${ }^{(21)}$.

En este estudio se identificó que no existe relación entre la competencia para el cuidado en el hogar de la persona con cáncer en quimioterapia y su cuidador familiar, hallazgo que permite inferir que, aunque existe el vínculo estrecho, las competencias para el cuidado se desarrollan de forma independiente. Sin embargo, es de resaltar que en nuestra investigación la competencia de la diada paciente-cuidador reportó niveles muy similares, lo que supone la pertinencia de diseñar intervenciones con enfoque sobre la diada para fortalecer vínculos de mutualidad y reciprocidad que permitirán mejor afrontamiento de esta situación y aprender juntos en pro de su bienestar ${ }^{(27)}$. Ahora bien, a nivel nacional otros estudios han evidenciado que los cuidadores no cuentan con niveles óptimos para desarrollar el rol que asumen ${ }^{(25)}$, las intervenciones que se han de generar en lograr un nivel de competencia óptimo requerirán de mayor participación y desarrollo de planes de egreso fomentando el traspaso de la información ${ }^{(28,29)}$, educación, entrenamiento y seguimiento con el fin de promover calidad y continuidad del cuidado tal como lo evidenció un estudio en adultos mayores ${ }^{(30)}$ y personas con cáncer de pulmón ${ }^{(31)}$.

Este estudio genera una orientación para abordar la competencia del cuidador y de la persona con cáncer en tratamiento con quimioterapia, como un fenómeno integral que se fundamenta en la consolidación de un sujeto de cuidado denominado diada.

Futuras investigaciones deben incluir la variable carga del cuidador como indicador de resultado de la competencia para el cuidado en el hogar dentro de los programas de alta; se requiere diseñar estudios de intervención dirigidas a las personas con cáncer y sus cuidadores que fortalezcan la competencia y permitan continuar con los cuidados a nivel domiciliario generando una transición del hospital al hogar adecuadas. Abordajes cualitativos identificando la experiencia de la diada en las unidades de quimioterapia son necesarios para determinar otras dimensiones que pueden estar relacionadas con los procesos y significados que se atribuyen a esta condición de salud.

\section{Referencias bibliográficas}

1. Organización Mundial de la Salud. Enfermedades no transmisibles y promoción de la salud www.paho.org. [Actualizado 25 de septiembre de 2014; Acceso 25 de abril de 2015] Disponible en: http://www.paho.org/gut/index.php?option=com content\&view $=$ article \&id $=589$

2. Cancer.net. Tratamiento contra el cáncer. [Actualizado 2005-2014; Acceso 10 de Abril de 2015] Disponible en: http://www.cancer.net/es/desplazarse-por-atenci\%C3\%B3ndel-c $\% \mathrm{C} 3 \% \mathrm{~A} 1$ ncer/el-c $\% \mathrm{C} 3 \% \mathrm{~A} 1$ ncer-en-adultos-mayores/tratamiento-contra-el$\mathrm{c} \% \mathrm{C} 3 \% \mathrm{~A} 1 \mathrm{ncer}$

3. Chaparro Díaz L. Trascender en un "Vínculo especial" de cuidado: El paso de lo evidente a lo intangible. [2009; Acceso 1 de enero de 2015]. Disponible en: www.bdigital.unal. edu.co

4. Pallett PA conceptual framework for studying family caregiver burden in Alzheimer's Type Dementia. Image: J Nurs Scholarsh 1990, 52-58

5. Schumacher KL. Reconceptualizing family caregiving: family-based illness care during chemotherapy. Res Nurs Health1996;19:261-71. 
6. Baker E, Wellman N. Nutrition concerns in discharge planning for older adults: A need for multidisciplinary collaboration. J Am Diet Assoc 2005;105:603-7. Doi: 10.1016/j. jada.2005.01.006

7. Carrillo GM, Sánchez Herrera B, Arias EM. Validación de un instrumento para la evaluación de la competencia de cuidado en el hogar del cuidado familiar de una persona con enfermedad crónica. Investigación y educación en enfermería. 2015;33:449-55. Doi:10.17533/udea.iee.v33n2a08

8. Chaparro L, Sánchez B, Carrillo GM. Encuesta de caracterización del cuidado de la diada cuidador familiar - persona con enfermedad crónica. Rev Cien Cuidad 2014;11:31-45

9. Carrillo-Gonzalez G, Barreto-Osorio R, Arboleda L, Gutiérrez-Lesmes O, Melo B, Ortiz T. Competencia para cuidar en el hogar de personas con enfermedad crónica y sus cuidadores en Colombia. Rev Fac Med 2015;63:665-75

10. Aldana E, Barrera S, Rodriguez K, Gómez O, Carrillo GM. Competencia para el cuidado (CUIDAR) en el hogar de personas con enfermedad renal crónica en hemodiálisis. Enferm Nefrol 2016;19:265-273, Doi:10.4321/S2254-28842016000300009

11. World Medical Association. Declaración de Helsinki de la AMM - Principios éticos para las investigaciones médicas en seres humanos. [Actualizado 2008; Acceso 15 de agosto de 2015]. Disponible en: http://www.wma.net/es/30publications/10policies/b3/

12. Kenneth John Ryan, et al. U.S. Department of health and Human Services. The Belmont Report. [Actualizado 1979; Acceso 15 de agosto de 2015]. Disponible en: http://www. hhs.gov/ohrp/humansubjects/guidance/belmont.html

13. CIOMS (2002). Pautas Éticas Internacionales para la Investigación Biomédica en Seres Humanos. [Acceso 1 de enero de 2017]. Disponible en: En: http:/www.cioms.ch/ publications/guidelines/pautas_eticas_internacionales.htm.

14. Ministerio de Salud y protección social. Resolución Número 8430 de 1993 [Actualizado 4 de Octubre 2013; Acceso 15 de agosto de 2015]. Disponible en: https:/www.minsalud. gov.co/Normatividad/RESOLUCION\%208430\%20DE\%201993.pdf

15. Tribunal Departamental Ético de Enfermería. Ley 266 de 1996. Ley 911 de 2004 “ Ley Deontológica para el ejercicio de la Profesión de Enfermería en Colombia. [Actualizado 2004; Acceso 15 de agosto de 2015]. Disponible en: http://www.tribdepetico.com/ ley_911.html

16. Universidad Nacional de Colombia. Legal.unal.edu.co [Actualizado 03 de diciembre de 2003; Acceso 2 de septiembre de 2015]. Disponible en: http://www.legal.unal.edu.co/ sisjurun/normas/Norma1.jsp?i=34248

17. Castañeda A. Calidad de vida y adherencia al tratamiento de personas con enfermedad crónica oncológica. Cuidarte 2015;6:906-13. Doi:10.15649/cuidarte.v6i1.146

18. Carrillo GM, Sánchez B, Barrera L. Habilidad de cuidado de cuidadores familiares de niños y adultos con enfermedad crónica. Index Enferm 2014;23:129-33. Doi: 10.4321/ S1132-12962014000200003

19. Puerto Pedraza HM, Carrillo González GM. Calidad de vida y soporte social percibido en los cuidadores familiares de personas en tratamiento contra el cáncer. Rev Univ Ind Santander Salud 2015;47:125-36

20. Krikorian A, Vélez MC, González O, Palacio C, Vargas J. La experiencia de sufrimiento en cuidadores principales de pacientes con dolor oncológico y no oncológico. Rev Enferm 2010; 28: 13-20

21. Barrera L, Campos MS; Gallardo K, Coral RC, Hernandez A. Soporte social percibido por las personas con enfermedad crónica y sus cuidadores familiares en cinco macro regiones geográficas de Colombia. Rev Univ Salud 2016;18:102-12 
22. Galvis CR, Pinzón ML, Romero E. Comparación entre la habilidad de cuidado de cuidadores de personas en situación de enfermedad crónica en Villavicencio, Meta. Av Enferm 2004;22:4-26

23. Breinbauer KH, Vásquez VH, Mayanz S, Guerra C, Millán K. Validación en Chile de la Escala de Sobrecarga del Cuidador de Zarit en sus versiones original y abreviada. Rev Méd Chile 2009;137:657-65. Doi:10.4067/S0034-98872009000500009

24. Ávila-Toscano JH, Vergara Mercado M. Calidad de vida en cuidadores informales de personas con enfermedades crónicas. Aquichan 2014);4: 417-29

25. Ramírez M. Habilidad del cuidado de los cuidadores principales de pacientes con cáncer gastrointestinal. Av Enferm 2005;13:7-17.

26. Blasco $\mathrm{T}$, Inglés N. Calidad de vida y adaptación a la enfermedad en pacientes de cáncer durante el tratamiento de quimioterapia. [Actualizado 1997; Acceso 10 marzo 2015] Disponible en: http://www.raco.cat/index.php/anuariopsicologia/article/ viewFile/61347/88771.

27. Hernández NE, Moreno CM, Barragán JA. Necesidades de cuidado de la díada cuidadorpersona: expectativa de cambio en intervenciones de enfermería. Rev Cuid 2014;5:74856. Doi:10.15649/cuidarte.v5i2.87

28. Sullivan BJ, Marcuccilli L, Sloan R, Gradus-Pizlo I, Bakas T, Jung M, et al. Competence, compassion, and care of the self: Family caregiving needs and concerns in heart failure. $\mathrm{J}$ Cardiovasc Nurs 2016;31: 209-14. Doi: 10.1097/JCN.0000000000000241.

29. Brockow K, Schallmayer S, Beyer K, Biedermann T, Fischer J, Gebert N, et al. Effects of a structured educational intervention on knowledge and emergency management in patients at risk for anaphylaxis. Allergy 2015;70:227-35. Doi: 10.1111/all.12548.

30. Zea Herrera MC, Torres Marín B. Adultos mayores dependientes hospitalizados: la transición del cuidado. Invest Educ Enferm 2007;25:40-9

31. Badr H, Smith CB, Goldstein NE, Gomez J, Redd WH. Dyadic psychosocial intervention for advanced lung cancer patients and their family caregivers: Results of a randomized pilot trial. Cancer 2015;121:150-8. Doi: 10.1002/cncr.29009. 